\title{
Photoplethysmogram Modeling during Paroxysmal Atrial Fibrillation: Detector Evaluation
}

\author{
Andrius Sološenko ${ }^{1}$, Andrius Petrènas ${ }^{1}$, Vaidotas Marozas ${ }^{1}$, Leif Sörnmo $^{2}$ \\ ${ }^{1}$ Biomedical Engineering Institute, Kaunas University of Technology, Kaunas, Lithuania \\ ${ }^{2}$ Department of Biomedical Engineering and Center of Integrative Electrocardiology, Lund \\ University, Lund, Sweden
}

\begin{abstract}
A phenomenological model for simulating photoplethysmogram $(P P G)$ during paroxysmal atrial fibrillation $(A F)$ is proposed. A PPG pulse is modeled by combining a lognormal and two Gaussian waveforms. Continuous PPG signals are produced by placing and connecting individual pulses according to the RR interval pattern extracted from annotated ECG signals. This paper presents a practical application of the proposed model for studying the performance of an RR-based AF detector. Physionet databases containing AF episodes serve as a basis for modeling $P P G$ signals. Detection performance was tested for different signal-to-noise ratios (SNRs), ranging from 0 to $30 \mathrm{~dB}$. The results show that an SNR of at least $15 \mathrm{~dB}$ is required to ensure adequate performance. Considering the lack of annotated, public PPG databases with arrhythmias, the modeling of realistic PPGs based on annotated ECG signals should facilitate the development and testing of PPGbased detectors.
\end{abstract}

\section{Introduction}

Although different technologies have been developed for atrial fibrillation (AF) detection, only ECG-based Holter monitors and event recorders are used in clinical routine. Since AF may be asymptomatic [1], patientfriendly screening technologies are highly desirable so that $\mathrm{AF}$ can be detected at an early stage. The emerging handheld ECG recorders are particularly easy to use, however, a short term ECG $(<1 \mathrm{~min})$ is not enough to detect paroxysmal AF. Therefore, it is essential to develop unobtrusive screening technologies capable of monitoring longer periods of time (i.e., up to months).

Photoplethysmography has been successfully applied to AF detection by employing a smartphone's camera [2-4]. However, the photoplethysmogram (PPG) is recorded only for short periods of time. With an increasing number of commercially available wristwatches or bracelet-type de- vices, capable of acquiring PPG continuously, it is a possible to unobtrusively screen AF for an unlimited period of time.

The lack of guidelines for PPG-based arrhythmia diagnosis implies that the annotation of arrhythmias in the PPG has to be based on the simultaneously acquired ECG. Since this process is time-consuming and very costly for day-long signals, simulated PPG signals can compensate for the lack of available PPG databases. Such databases are urgently needed to facilitate the ongoing development and testing of PPG-based AF detectors. To the best of our knowledge, only the Physionet MIMIC, MIMIC II and the University of Queensland Vital Signs Dataset databases have synchronously recorded, however, unannotated PPG and ECG signals. Given that Physionet provides many ECG databases with annotated AF episodes (i.e., MIT-BIH Arrhythmia, MIT-BIH Atrial Fibrillation, MITBIH Long-Term Atrial Fibrillation), these public databases could be employed to produce simulated PPG signals.

This paper presents a practical application of a phenomenological model for simulating PPG signals during paroxysmal AF. The model, first introduced in [5], uses the RR interval series as a basis for generating a PPG signal. In the present study, we exemplify the practical value of the proposed simulation model by studying AF detection performance on PPG signals, generated from RR intervals taken from public ECG databases.

\section{Simulation Model}

The RR interval series, obtained from an annotated ECG recording, serves as the input to the proposed simulation model, see Fig. 1. The model consists of two main parts, namely, modeling of a single PPG pulse and concatenation of pulses into a connected signal.

A PPG pulse is modeled as a linear combination of three functions-one log-normal waveform and two Gaussians-together accounting for direct and reflected 


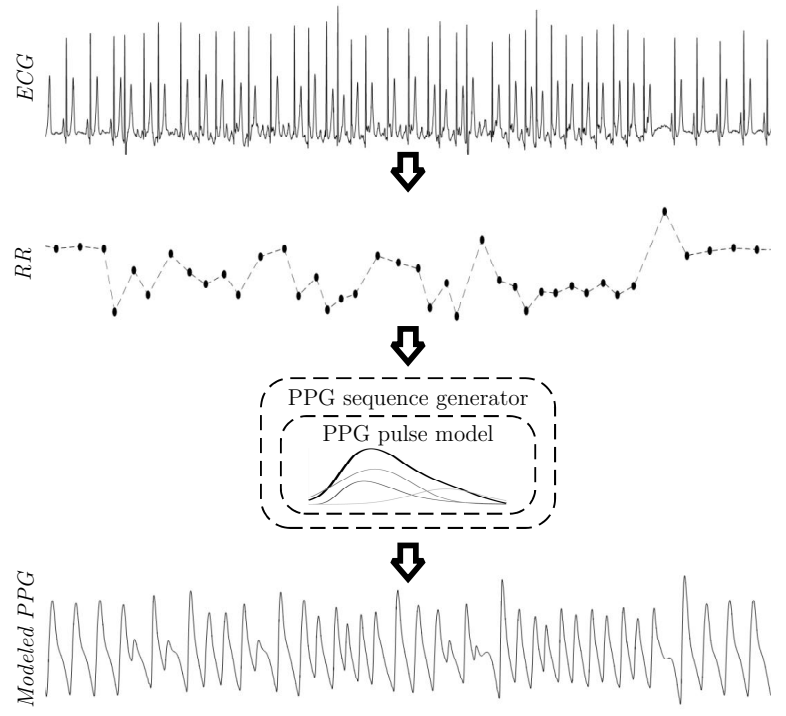

Figure 1. Block diagram of the proposed model for simulation of PPG signals during paroxysmal AF.

pulse waves [6]. The log-normal function is defined as

$$
\varphi_{1}\left(t ; m, \sigma_{1}\right)= \begin{cases}\frac{1}{t \sqrt{2 \pi \sigma_{1}^{2}}} e^{-\frac{(\ln (t / m))^{2}}{2 \sigma_{1}^{2}}}, & t>0, \\ 0, & t \leq 0\end{cases}
$$

where $t$ is time, $m$ is a scale parameter, and $\sigma_{1}^{2}$ is a shape parameter. The Gaussian waveform is defined by

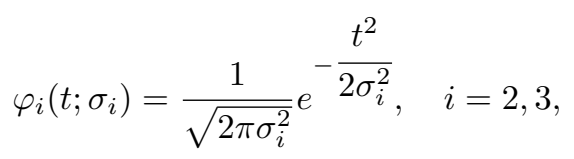

where $\sigma_{i}^{2}$ is a width parameter. Then, the PPG pulse is modeled as a linear combination of weighted, time-shifted, and time-scaled versions of $\varphi_{1}\left(t ; m, \sigma_{1}\right), \varphi_{2}\left(t ; \sigma_{2}\right)$, and $\varphi_{3}\left(t ; \sigma_{3}\right)$, i.e.,

$\varphi(t ; \boldsymbol{\theta})=w_{1} \varphi_{1}\left(t-\tau_{1} ; m, \sigma_{1}\right)+\sum_{i=2}^{3} w_{i} \varphi_{i}\left(t-\tau_{i} ; \sigma_{i}\right)+a$,

where $a$ denotes DC offset. For convenience, all model parameters are merged into the vector

$$
\boldsymbol{\theta}=\left[w_{1}, w_{2}, w_{3}, \tau_{1}, \tau_{2}, \tau_{3}, m, \sigma_{1}, \sigma_{2}, \sigma_{3}, a\right] .
$$

The parameters of the PPG pulse are estimated by nonlinear least squares fitting,

$$
J(\boldsymbol{\theta})=\int_{-\infty}^{\infty}(y(t)-\varphi(t ; \boldsymbol{\theta}))^{2} d t
$$

$$
\hat{\boldsymbol{\theta}}=\arg \min _{\boldsymbol{\theta}} J(\boldsymbol{\theta}),
$$

where $\hat{\boldsymbol{\theta}}$ is the vector minimizing the difference between the PPG pulse template $y(t)$, a pulse extracted from the real PPG signal, and the modeled PPG pulse $\varphi(t ; \boldsymbol{\theta})$. Prior to minimization, each PPG template is normalized to unit amplitude. A PPG pulse is composed of a systolic part and a diastolic part, where the width of each part depends on the adjacent RR intervals. For the $k$ :th pulse, two time scale factors are introduced, both inversely proportional to RR intervals,

$$
\begin{gathered}
\beta_{s, k}= \begin{cases}\frac{1}{r_{k}}, & \frac{r_{k}}{r_{k-1}} \leq \eta_{0}, \\
\frac{1}{r_{k-1}}, & \text { otherwise, }\end{cases} \\
\beta_{d, k}= \begin{cases}\frac{1}{r_{k+1}}, & \frac{r_{k}}{r_{k-1}} \leq \eta_{0}, \\
\frac{1}{r_{k}}, & \text { otherwise },\end{cases}
\end{gathered}
$$

where $r_{k}$ denotes the RR interval preceding the $k$ :th pulse. The threshold $\eta_{0}=0.8$ determines whether the current beat is premature.

Another factor is the PPG pulse amplitude related to ventricular filling time and, accordingly, to the length of the RR interval. For example, in sinus rhythm, the ventricular filling time does not change much from beat to beat, leading to negligible pulse amplitude variations. On the contrary, a premature beat causes diastole to be shorter, reducing the amplitude of the resulting PPG pulse.

The amplitude of a PPG pulse is assumed to be proportional to $r_{k}$, unless the beat is premature when the relationship between the length of the current RR interval and the diastolic period can be characterized by an exponential function. The pulse amplitude is given by

$$
\alpha_{k}= \begin{cases}0.58 \cdot r_{k}^{1.32}, & \frac{r_{k}}{r_{k-1}} \leq \eta_{0} \text { or } \frac{r_{k+1}}{r_{k}} \geq \eta_{1}, \\ r_{k}, & \text { otherwise. }\end{cases}
$$

where the threshold $\eta_{1}=1.4$ determines whether the subsequent beat is premature, thus allowing the decrease in amplitude.

The $k$ :th PPG pulse, denoted $s_{a, k}(t)$, is put into context by scaling the amplitude of $\varphi(t ; \hat{\boldsymbol{\theta}})$ with $\alpha_{k}$ and the width with either $\beta_{s, k}$ or $\beta_{d, k}$ :

$$
s_{a, k}(t)= \begin{cases}\alpha_{k} \cdot \varphi\left(\beta_{s, k}\left(t-t_{p}\right) ; \hat{\boldsymbol{\theta}}\right), & -\infty<t<t_{p}, \\ \alpha_{k} \cdot \varphi\left(\beta_{d, k}\left(t-t_{p}\right) ; \hat{\boldsymbol{\theta}}\right), & t_{p} \leq t<\infty,\end{cases}
$$

where $t_{p}$ is the time for the largest positive peak in $\varphi(t ; \hat{\boldsymbol{\theta}})$. Finally, $s_{a, k}(t)$ is sampled at a chosen rate, resulting in the signal $s_{k}(n)$. 

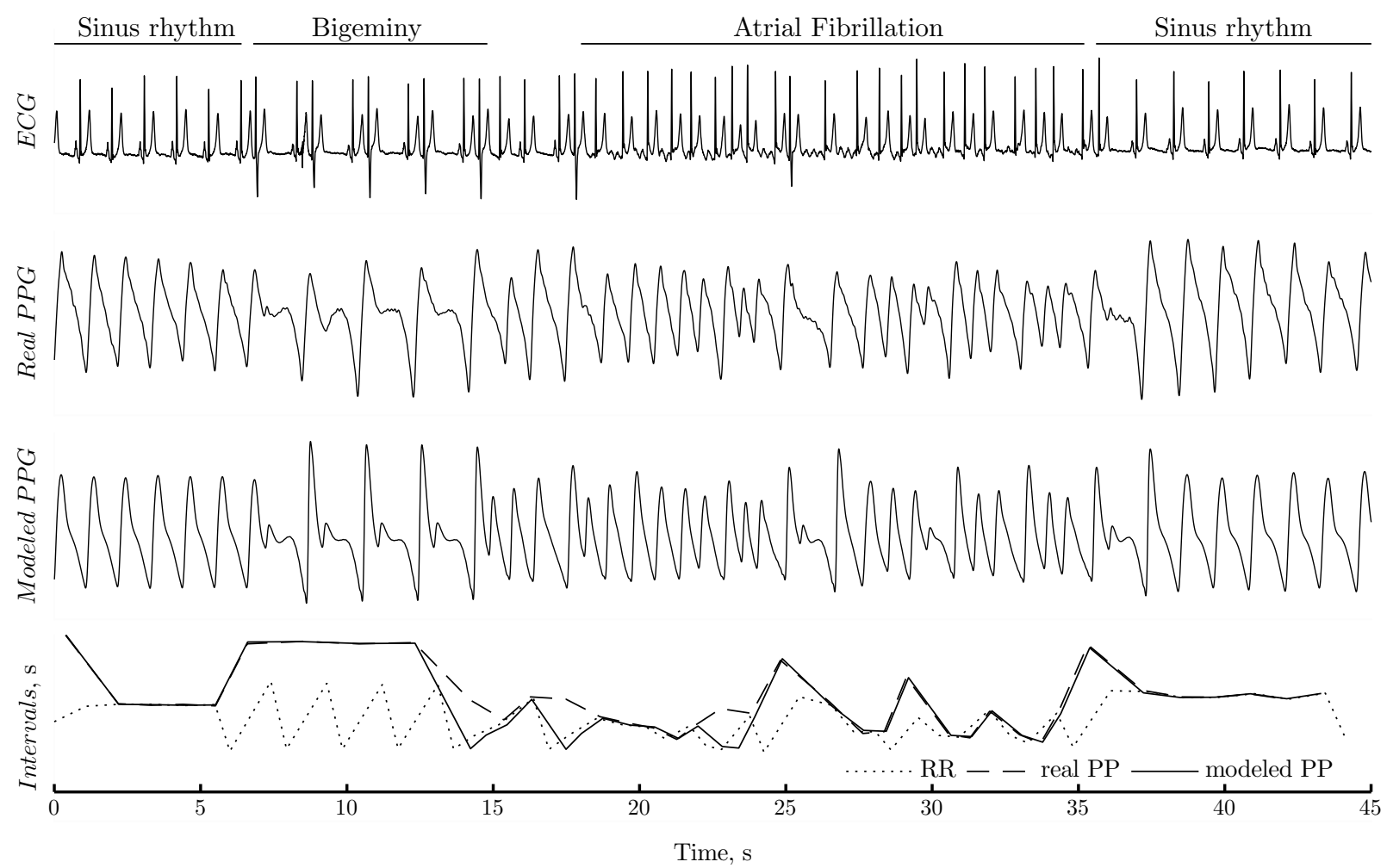

Figure 2. Synchronously recorded ECG and PPG signals with paroxysmal AF compared to the simulated PPG signal. The bottom row shows RR intervals and peak-to-peak (PP) intervals of the real and modeled PPG signals, respectively.

A connected, discrete-time model signal $x(n)$ is generated by placing the contextualized PPG pulses at the heartbeat occurrence times $\delta_{k}$, obtained from the RR interval series, and adding noise $v(n)$,

$$
x(n)=\sum_{k=1}^{K} s_{k}\left(n-\delta_{k}\right)+v(n),
$$

where $K$ is the number of pulses in the connected signal.

The noise $v(n)$ is generated by filtering white noise, where the filter is determined by the spectral properties of motion artifacts extracted from PPG signals in the MIMIC database. Using the above simulation model, a noise-free signal is generated from the RR intervals of the segment with the artifact. The artifact is extracted by cancelling the model signal from the observed signal using a normalized least-mean squares (NLMS) filter. Then, the extracted artifact serves as the desired input to the NLMS filter, whereas white noise is reference input. The filter output is a signal resembling the extracted artifact. The resulting impulse response of the NLMS filter is the used for producing $v(n)$.

Example of real and modeled PPG signals during paroxysmal AF, preceded by an episode of bigeminy, is presented in Fig. 2.

\section{Peak and AF detection}

The modeled PPG signal, subject to AF detection, is preprocessed using an FIR bandpass filter with cut-off frequencies at 0.5 and $5 \mathrm{~Hz}$ to remove noise components. Then, a real-time peak detector, based on [7], is applied to the preprocessed signal so that the intervals between successive pulses can be determined and used as input to the AF detector.

The significance of the PPG model is studied in terms of detection performance at different signal-to-noise ratios (SNRs). A low-complexity AF detector based on RR interval information, designed to detect brief episodes $(<30 \mathrm{~s})$, is studied [8]. Important features of the detector is the short sliding detection window ( 8 beats) and the low-power consumption, making the detector particularly well-suited for implementation in a wearable system.

\section{Databases and performance measures}

PPG signals were simulated using ECGs of the MITBIH Atrial Fibrillation (AFDB), the MIT-BIH Long-Term Atrial Fibrillation (LTAFDB), and the MIT-BIH Arrhythmia (MITDB) databases [9]. The signals were simulated at SNRs ranging from 0 to $30 \mathrm{~dB}$.

The AF detection performance is evaluated in terms of 
sensitivity $(S e)$ and specificity $(S p)$. Sensitivity is defined by the number of correctly detected AF beats divided by the total number of AF beats. Specificity is defined as the number of correctly detected non-AF beats divided by the total number of non-AF beats.

\section{Results}

Figure 3 shows detection performance as a function of SNR. The performance of the AF detector is largely dependent on the accuracy of the PPG pulse detector since the series with peak-to-peak intervals serves as the input to the AF detector. The number of false positives increases considerably at lower SNRs due to incorrect peak detection, whereas the increase in $S e$ at low SNRs is due to detection of noise-induced peaks, causing irregular pulseto-pulse intervals.
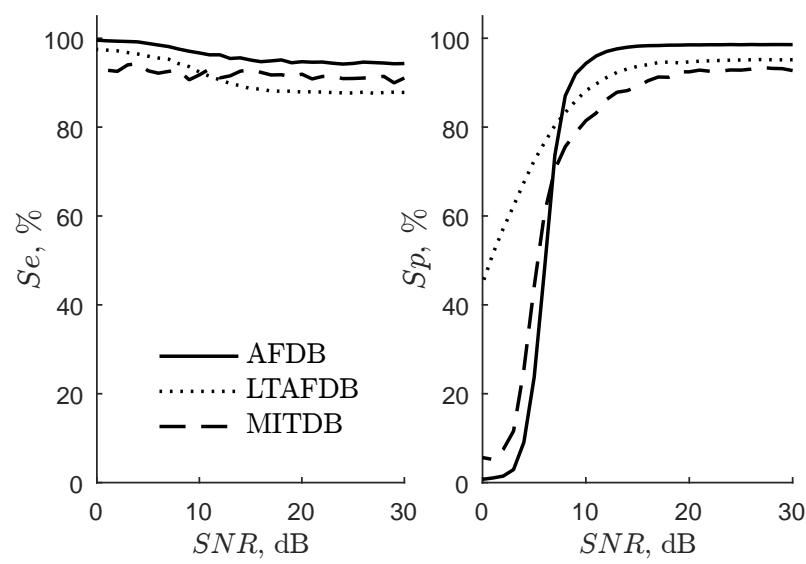

Figure 3. AF detection performance on PPG databases, generated using the AFDB, LTAFDB, and MITDB.

When studying the AF detector on AFDB, Se and $S p$ are found to be $97.1 \%$ and $98.3 \%$, respectively [8]. To achieve the same performance on the simulated PPG database, SNR levels of $(>15 \mathrm{~dB})$ are required.

\section{Conclusions}

A phenomenological model for simulating PPG signals during paroxysmal AF is introduced. The possibility to simulate connected PPG signals at different SNRs is valuable when developing and testing PPG-based AF detectors.

\section{Acknowledgements}

This work was supported by the Research Council of Lithuania (Agreement No. MIP088/15).

\section{References}

[1] Lowres N, Neubeck L, Redfern J, Freedman SB. Screening to identify unknown atrial fibrillation. A systematic review. Thromb Haemost Aug 2013;110(2):213-222.

[2] Lewis M, Parker D, Weston C, Bowes M. Screening for atrial fibrillation: sensitivity and specificity of a new methodology. Br J Gen Pract Jan 2011;61(582):38-39.

[3] Lee J, Reyes B, McManus D, Mathias O, Chon K. Atrial fibrillation detection using an iPhone 4S. IEEE Trans Biomed Eng Jan 2013;60(1):203-206. ISSN 0018-9294.

[4] Freedman B. Screening for atrial fibrillation using a smartphone: Is there an app for that? J Am Heart Assoc Jul 2016; $5(7)$.

[5] Sološenko A, Petrènas A, Marozas V, Sörnmo L. Modeling of the photoplethysmogram during atrial fibrillation. Comput Biol Med Jan 2017;81:130-138. ISSN 0010-4825.

[6] Baruch MC, Warburton DE, Bredin SS, Cote A, Gerdt DW, Adkins CM. Pulse decomposition analysis of the digital arterial pulse during hemorrhage simulation. Nonlin Biomed Phys 2011;5(1):1-15. ISSN 1753-4631.

[7] Aboy M, McNames J, Thong T, Tsunami D, Ellenby M, Goldstein B. An automatic beat detection algorithm for pressure signals. IEEE Trans Biomed Eng Oct 2005; 52(10):1662-1670. ISSN 0018-9294.

[8] Petrènas A, Marozas V, Sörnmo L. Low-complexity detection of atrial fibrillation in continuous long-term monitoring. Comput Biol Med 2015;65:184 - 191. ISSN 0010-4825.

[9] Goldberger AL, Amaral LAN, Glass L, Hausdorff JM, Ivanov PC, Mark RG, Mietus JE, Moody GB, Peng CK, Stanley HE. PhysioBank, Physiotoolkit, and Physionet: Components of a new research resource for complex physiologic signals. Circulation 2000;101(23):215-220.

Address for correspondence:

Name: Andrius Sološenko

Full postal address: Biomedical Engineering Institute, Kaunas University of Technology, K. Barsausko st. 59, LT-51423 Kaunas, Lithuania.

E-mail address: andrius.solosenko@ktu.lt 